\title{
Retrospective study of single vitrified-warmed blastocyst transfer cycles according to the presence of morphokinetic variables
}

\author{
Yong Soo Hur', Eun Kyung Ryu', Chang Seop Hyun², Seong Ho Yang ${ }^{3}$, San Hyun Yoon ${ }^{4}$, Kyung Sil Lim', Won Don Lee', Jin Ho Lim¹ \\ 'Maria Fertility Hospital, Seoul; ${ }^{2}$ Pyungchon Maria Fertility Clinic, Anyang; ${ }^{3}$ Maria Plus Fertility Hospital, Seoul; ${ }^{4}$ Maria Research Center, Seoul, Korea
}

This study retrospectively assessed whether time-lapse data relating to developmental timing and morphology were associated with clinical outcomes, with the eventual goal of using morphokinetic variables to select embryos prospectively for cryopreservation. In this study, we examined the clinical outcomes of single vitrified-warmed blastocyst transfer cycles that were cultured in a time-lapse incubation system. The morphokinetic variables included uneven pronuclei, an uneven blastomere, multinucleation, and direct, rapid, and irregular division. A total of 164 single vitrified-warmed blastocyst transfer cycles were analyzed (102 cycles of regularly developed blastocysts and 62 cycles of blastocysts with morphokinetic variables). No significant differences in the age of females or the standard blastocyst morphology were found between these two groups. The regularly developed blastocysts showed significantly higher implantation and clinical pregnancy rates than the blastocysts exhibiting morphokinetic variables ( $30.4 \%$ vs. $9.7 \%$ and $37.3 \%$ vs. $14.5 \%$, respectively; $p<0.01)$. The blastocysts that exhibited morphokinetic variables showed different mean development times compared with the regularly developed blastocysts. Although morphokinetic variables are known to have fatal impacts on embryonic development, a considerable number of embryos developed to the blastocyst stage. Morphokinetic variables had negative effects on the implantation and clinical pregnancy rates in vitrified-warmed blastocyst transfer cycles. These findings suggest that blastocysts cultured in a time-lapse incubation system should be considered for selective cryopreservation according to morphokinetic variables.

Keywords: Blastocyst; Single embryo transfer; Time-lapse

Time-lapse monitoring and incubation systems have enabled embryologists to observe the development of embryos continuously and more precisely. Time-lapse incubation systems allow the identification of viable embryos based on morphokinetics, which refers to the timing of embryo development associated with the implantation potential of transferred embryos. Recently, time-lapse incubation systems have been used to select viable embryos for single embryo transfer [1]. In such systems, an effort is made to select a single viable

Received: Sep 19, 2017· Revised: Nov 29, 2017 · Accepted: Jan 15, 2018 Corresponding author: Yong Soo Hur

Maria Fertility Hospital, 20 Cheonho-daero, Dongdaemun-gu, Seoul 02586, Korea

Tel: +82-2-2250-5572 Fax: +82-2-2250-5585 E-mail: geaher@mariababy.com

This is an Open Access article distributed under the terms of the Creative Commons Attribution Non-Commercial License (http://creativecommons.org/licenses/by-nc/4.0/) which permits unrestricted non-commercial use, distribution, and reproduction in any medium, provided the original work is properly cited. embryo for blastocyst culture and transfer. Generally, it is preferable to transfer blastocysts rather than cleavage-stage embryos because day 5 blastocysts are more physiologically suitable for implantation in a uterus with normal unstimulated cycles, and the extended culture time allows the selection of higher-quality embryos [2]. The determination of the quality of blastocysts cultured in conventional culture systems depends on the morphology-dependent selection method, which is determined by the quality of the inner cell mass, trophectoderm cells, and the state of expansion [3]. The viability of frozen-thawed embryos also depends upon the morphological quality of the preimplantation embryos before freezing and after thawing [4].

Our laboratory uses a time-lapse incubation system for fresh cycles, and transferred embryos are determined to be viable using morphokinetic variables and a standard embryo scoring system. However, 
surplus freezing-grade blastocysts are frozen and then thawed for embryo transfer without considering morphokinetic variables. This procedure is based on the belief that blastocyst development is a natural consequence of higher-quality embryo selection through extended culture. This procedure also involves overlooking morphokinetic variables and operating the cryopreservation program according to traditional practices. This study investigated the applicability of morphokinetic variables when thawing embryos that had been cultured in a time-lapse system for transfer.

Before the introduction of time-lapse incubation systems, intermittent observations to detect morphokinetic variables required exposing the embryo to an external environment, which has negative effects on embryo development [5]. However, the introduction of time-lapse incubation systems has enabled various morphokinetic variables to be observed during embryo development without causing embryo damage. Morphokinetic variables, such as fragmentation, multinucleation, uneven pronuclei (PN), and an uneven blastomere, have been observed in conventional incubation systems. Adverse effects on implantation and pregnancy rates due to these morphokinetic variables have been previously reported using conventional incubation systems [6-10]. In addition to the above factors, the effects on clinical outcomes of reverse division, direct division, and rapid division, which are not easily observed in conventional culture systems, are also being actively studied.

This study retrospectively assessed whether time-lapse data relating to developmental timing and morphology were associated with clinical outcomes, with the eventual goal of using morphokinetic variables to select embryos prospectively for cryopreservation. In this study, we examined the clinical outcomes of single vitrified-warmed blastocyst transfer cycles that had been cultured in a time-lapse incubation system. A total of 164 single vitrified-warmed blastocyst transfer cycles were performed at the Maria Fertility Hospital and Pyungchon Maria Fertility Clinic from January 2014 to October 2016. Single vitrified-warmed blastocyst transfer cycles were divided into two groups: regularly developed blastocysts (102 cycles) and blastocysts exhibiting morphokinetic variables (62 cycles). Sixty-two cycles of blastocysts exhibiting irregular morphokinetics were analyzed in terms of morphokinetic variables such as multinucleation, uneven $\mathrm{PN}$, an uneven blastomere, reverse division, direct division, and rapid division. This study was approved by the Institutional Review Board of Maria Fertility Hospital (IRB No. 2017-001).

A gonadotropin-releasing hormone agonist long protocol or a gonadotropin-releasing hormone antagonist protocol was used in the fresh cycles. Oocyte retrieval was performed 36 hours after human chorionic gonadotropin injection. Intracytoplasmic sperm injection was performed in denuded mature oocytes. Oocytes were then placed in pre-equilibrated dishes (EmbryoSlide; Unisense FertiliTech,
Aarhus, Denmark) and fertilization was assessed 15-18 hours after insemination, based on the presence of two PN. Embryos were monitored in a time-lapse incubation system (EmbryoScope, Unisense FertiliTech). Embryos were cultured in $6 \% \mathrm{CO}_{2}, 5 \% \mathrm{O}_{2}$, and $89 \% \mathrm{~N}_{2}$ at $37^{\circ} \mathrm{C}$ in Sydney IVF Cleavage Medium (COOK, Brisbane, Australia) for 48 hours. Embryos were selected for transfer and surplus embryos were cultured in Sydney IVF Blastocyst Medium (COOK) for blastocyst development. The quality of the inner cell mass, trophectoderm, and the state of expansion was evaluated according to the blastocyst scoring system [11], and blastocysts graded as 3BB or higher were defined as freezing-grade and cryopreserved.

Vitrification and thawing processes were performed as described previously [12]. In thawed blastocysts, the inner cell mass and trophectoderm cells were evaluated according to the blastocyst scoring system, and blastocysts graded CC or higher were used for embryo transfer.

In this study, the morphokinetic variables observed during timelapse culture included uneven PN, an uneven blastomere, multinucleation, direct division, irregular division, and rapid division. Uneven $\mathrm{PN}$ and an uneven blastomere were defined as the size of the nucleus and blastomere differing by more than $30 \%$. Direct division reflected an abnormal length of the cell cycle, such as the abrupt cleavage of embryos from two to three cells in less than 5 hours. The category of irregular division included all types of irregular division, including reverse division. Although most morphokinetic variables had a fatal impact upon embryo development, some embryos developed to the blastocyst stage during time-lapse culture. These blastocysts were frozen and used for thawing cycles if the blastocyst quality was defined as freezing-grade. Blastocyst morphology was observed prior to transfer. Morphokinetic observations ( $t_{\text {PN faded, }}$ time when both PN had faded; $\mathrm{t} 3$, time of cleavage to a 3-cell blastomere; $\mathrm{t5}$ : time of cleavage to a 5-cell blastomere; tSB, time of initiation of blastulation; cc2, duration of second cell cycle; s2, time of synchrony of the second cell cycle) were recorded in the EmbryoViewer workstation (Unisense FertiliTech) [13]. Time-lapse images were analyzed by one of the three senior researchers (YSH, EKR). Clinical pregnancy was confirmed by the presence of a gestational sac at 6-7 weeks. Implantation was confirmed by the presence of fetal heart activity at 12 weeks of pregnancy. Values were presented as the mean with standard deviation, or percentage. Between-group differences, including differences in the clinical pregnancy and implantation rates, were analyzed using the chi-square test. Differences were considered statistically significant if $p<0.01$.

No significant differences were found in the age of females or the standard blastocyst morphology between the regularly developed blastocysts and the blastocysts exhibiting morphokinetic variables. However, the implantation and clinical pregnancy rates of regularly 
developed blastocysts were significantly higher than the rates of blastocysts exhibiting morphokinetic variables (Table 1). The implantation and pregnancy rates were assessed according to the morphokinetic variables associated with the transferred blastocysts. Blastocysts exhibiting morphokinetic variables, such as uneven PN, multinucleation, and irregular division, had lower implantation and pregnancy rates than the regularly developed blastocysts. The morphokinetic variables observed during the cleavage stage, such as an uneven blastomere, direct division, and rapid division, had adverse effects on the implantation and clinical pregnancy rates. Regularly developed blastocysts shared common mean times, whereas blastocysts exhibiting morphokinetic variables did not show common morphokinetic features. Morphokinetic variables seemed to affect the embryo development rate, as the development rate varied depending on the morphokinetic variable. In particular, at the early cleavage stage, blastocysts exhibiting morphokinetic variables, such as direct and rapid division, had shorter mean times than regularly developed blastocysts (Table 2).

The results of this study can be summarized into three major points. First, although it is well known that most morphokinetic variables observed at an early stage have a fatal impact upon embryo development, many embryos capable of development into blastocysts were observed. The rate of regularly developed blastocysts and blastocysts exhibiting morphokinetic variables was high across the vitrified-warmed single blastocyst transfer cycles over a period of time. In conventional culture systems, it was believed that development into a blastocyst was a natural consequence of higher-quality embryo selection through extended culture. However, in the time-lapse incubation system, it was found that a nonnegligible number of embryos with morphokinetic variables at an early stage survived and developed into the blastocyst stage. In fact, the morphological differences between regularly developed blastocysts and blastocysts exhibiting morphokinetic variables were difficult to distinguish during the prefreezing and post-thaw processes. This may be a reason why high-

Table 1. Comparison of clinical outcomes according to the morphokinetic variables of the blastocysts

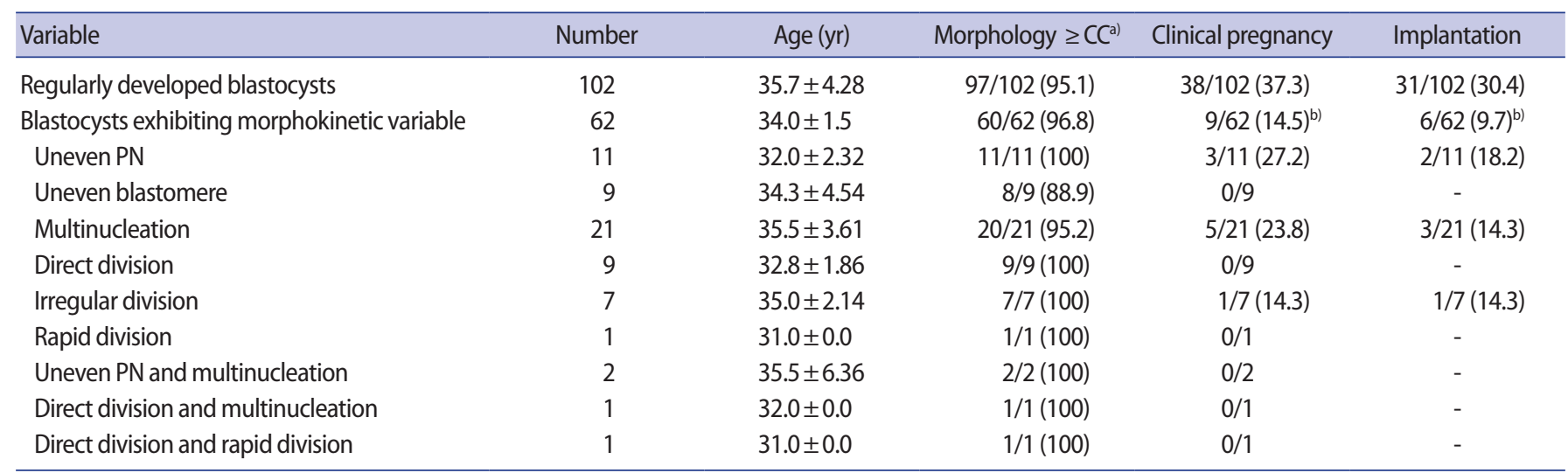

Values are presented as mean \pm standard deviation or number (\%).

$\mathrm{PN}$, pronuclei.

a) $\geq$ CC: more than the trophectoderm and inner cell mass grades using Gardner blastocyst grading system; ${ }^{\text {b) }} p<0.01$.

Table 2. Morphokinetic analysis of normally developed blastocysts and blastocysts exhibiting morphokinetic variables

\begin{tabular}{|c|c|c|c|c|c|c|c|}
\hline Variable & Number & $t_{\text {PN faded }}$ & t3 & t5 & $\mathrm{tSB}$ & $\mathrm{cc} 2(\mathrm{t} 3-\mathrm{t} 2)$ & $\mathrm{s} 2(\mathrm{t} 4-\mathrm{t} 3)$ \\
\hline Uneven PN & 11 & $25.3 \pm 4.1$ & $41.3 \pm 3.9$ & $58.0 \pm 6.5$ & $107.3 \pm 8.1$ & $12.4 \pm 0.7$ & $0.7 \pm 1.0$ \\
\hline Multinucleation & 21 & $25.9 \pm 3.6$ & $38.2 \pm 4.3$ & $53.3 \pm 5.4$ & $102.9 \pm 7.5$ & $9.8 \pm 4.2$ & $3.7 \pm 4.9$ \\
\hline Direct division & 9 & $26.0 \pm 1.7$ & $30.6 \pm 3.3$ & $40.3 \pm 2.8$ & $105.9 \pm 2.0$ & $2.1 \pm 1.7$ & $6.3 \pm 4.5$ \\
\hline Irregular division & 7 & $23.9 \pm 2.2$ & $34.7 \pm 4.3$ & $46.4 \pm 7.4$ & $100.1 \pm 6.9$ & $8.5 \pm 4.2$ & $6.7 \pm 7.3$ \\
\hline Uneven PN and multinucleation & 2 & $25.5 \pm 2.6$ & $38.9 \pm 3.3$ & $54.8 \pm 4.6$ & $112.9 \pm 1.0$ & $8.9 \pm 4.1$ & $1.0 \pm 0.7$ \\
\hline Direct division and multinucleation & 1 & $26.5 \pm 0.0$ & $43.7 \pm 0.0$ & $44.4 \pm 0.0$ & $96.5 \pm 0.0$ & $13.0 \pm 0.0$ & 0 \\
\hline Direct division and rapid division & 1 & $23.5 \pm 0.0$ & $29.3 \pm 0.0$ & $40.8 \pm 0.0$ & $104.7 \pm 0.0$ & 0 & $1.3 \pm 0.0$ \\
\hline
\end{tabular}

Values are presented as mean \pm standard deviation.

$t_{\mathrm{PN} \text { faded, }}$ time when both pronuclei had faded; $\mathrm{t}$, time of cleavage to a 3-cell blastomere; $\mathrm{t}$, time of cleavage to a 5-cell blastomere; $\mathrm{tSB}$, time of initiation of blastulation; $\mathrm{cc} 2$, duration of second cell cycle (t3-t2); s2, time of synchrony of the second cell cycle (t4-t3). 
quality vitrified-warmed blastocysts were not implanted in a conventional culture system that had no morphokinetic data.

Second, morphokinetic variables, such as uneven $\mathrm{PN}$, an uneven blastomere, multinucleation, direct division, irregular division, and rapid division, had negative effects on the implantation and clinical pregnancy rates in this study, as has been observed in fresh cycles. Adverse effects of these morphokinetic variables on implantation and pregnancy rates have previously been reported using conventional incubation systems [6-10]. In the current study, multinucleation and reverse division detected by the time-lapse incubation system were associated with lower implantation and clinical pregnancy rates in fresh cycles [14,15]. Earlier work likewise found that embryos with direct division and an uneven blastomere had significantly lower implantation rates than embryos exhibiting a normal cleavage pattern in fresh cycles [16]. Depending on the morphokinetic variables, the embryonic development rate changed, and the negative effects on implantation and pregnancy rates were somewhat different between this study and previous studies. Although the sample size of this study of single vitrified-warmed blastocyst transfer cycles was small, the adverse effects of morphokinetic variables were clear.

Third, it is necessary to consider selective freezing of embryos cultured using a time-lapse incubation system. This is because blastocysts with morphokinetic variables are not easy to implant even if they show good morphology after thawing. If more data are accumulated and the adverse effects on the implantation and pregnancy rate according to the type of morphokinetic variables become clear, the need for selective cryopreservation will arise. More studies and more discussion about the selective cryopreservation of embryos with morphokinetic variables are required.

\section{Conflict of interest}

No potential conflict of interest relevant to this article was reported.

\section{References}

1. Haikin Herzberger E, Ghetler Y, Tamir Yaniv R, Berkovitz A, Gonen 0 , Cohen I, et al. Time lapse microscopy is useful for elective single-embryo transfer. Gynecol Endocrinol 2016;32:816-18.

2. Papanikolaou EG, Kolibianakis EM, Tournaye H, Venetis CA, Fate$\mathrm{mi} \mathrm{H}$, Tarlatzis B, et al. Live birth rates after transfer of equal number of blastocysts or cleavage-stage embryos in IVF: a systematic review and meta-analysis. Hum Reprod 2008;23:91-9.

3. Gardner DK, Schoolcraft WB, Wagley L, Schlenker T, Stevens J, Hesla J. A prospective randomized trial of blastocyst culture and transfer in in-vitro fertilization. Hum Reprod 1998;13:3434-40.

4. Goto S, Kadowaki T, Tanaka S, Hashimoto H, Kokeguchi S, Shiotani M. Prediction of pregnancy rate by blastocyst morphological score and age, based on 1,488 single frozen-thawed blastocyst transfer cycles. Fertil Steril 2011;95:948-52.

5. Nakahara $T$, Iwase $A$, Goto $M$, Harata $T$, Suzuki $M$, lenaga $M$, et al. Evaluation of the safety of time-lapse observations for human embryos. J Assist Reprod Genet 2010;27:93-6.

6. Manor D, Drugan A, Stein D, Pillar M, Itskovitz-Eldor J. Unequal pronuclear size: a powerful predictor of embryonic chromosome anomalies. J Assist Reprod Genet 1999;16:385-9.

7. Alikani M, Cohen J, Tomkin G, Garrisi GJ, Mack C, Scott RT. Human embryo fragmentation in vitro and its implications for pregnancy and implantation. Fertil Steril 1999;71:836-42.

8. Hardarson T, Hanson C, Sjogren A, Lundin K. Human embryos with unevenly sized blastomeres have lower pregnancy and implantation rates: indications for aneuploidy and multinucleation. Hum Reprod 2001;16:313-8.

9. Van Royen E, Mangelschots K, Vercruyssen M, De Neubourg D, Valkenburg M, Ryckaert G, et al. Multinucleation in cleavage stage embryos. Hum Reprod 2003;18:1062-9.

10. Yakin K, Balaban B, Urman B. Impact of the presence of one or more multinucleated blastomeres on the developmental potential of the embryo to the blastocyst stage. Fertil Steril 2005;83: 243-5.

11. Gardner DK, Lane M, Stevens J, Schlenker T, Schoolcraft WB. Blastocyst score affects implantation and pregnancy outcome: towards a single blastocyst transfer. Fertil Steril 2000;73:1155-8.

12. Hur YS, Ryu EK, Song SH, Yoon SH, Lim KS, Lee WD, et al. A retrospective study of single frozen-thawed blastocyst transfer. Clin Exp Reprod Med 2016;43:106-11.

13. Meseguer M, Herrero J, Tejera A, Hilligsoe KM, Ramsing NB, Remohi J. The use of morphokinetics as a predictor of embryo implantation. Hum Reprod 2011;26:2658-71.

14. Liu Y, Chapple V, Roberts P, Matson P. Prevalence, consequence, and significance of reverse cleavage by human embryos viewed with the use of the Embryoscope time-lapse video system. Fertil Steril 2014;102:1295-300.e2.

15. Ergin EG, Caliskan E, Yalcinkaya E, Oztel Z, Cokelez K, Ozay A, et al. Frequency of embryo multinucleation detected by timelapse system and its impact on pregnancy outcome. Fertil Steril 2014;102:1029-33.e1.

16. Rubio I, Kuhlmann R, Agerholm I, Kirk J, Herrero J, Escriba MJ, et al. Limited implantation success of direct-cleaved human zygotes: a time-lapse study. Fertil Steril 2012;98:1458-63. 